\title{
Percepción de la calidad y satisfacción de la elección de los estudiantes de primer semestre en la Facultad de Ciencias Económicas de la Universidad Nacional de Colombia
}

Perception of the Quality and Satisfaction of the First-Semester Student Selection at the Faculty of Economic Sciences at Universidad Nacional de Colombia

Luz Alexandra Montoya R. (Colombia)

Universidad Nacional de Colombia lamontoyar@unal.edu.co
Iván Alonso Montoya R. (Colombia)

Universidad Nacional de Colombia iamontoyar@unal.edu.co

\section{Sandra Patricia Rojas (México) \\ Instituto Politécnico Nacional sprojasb@gmail.com}

\author{
Resumen \\ El presente documento presenta los \\ resultados de una investigación realizada \\ a 86 estudiantes de primer semestre en \\ la Facultad de Ciencias Económicas de
}

\begin{abstract}
This documents presents the results of an investigation carried out with 86 students of first semester belonging to the Faculty of Economic Sciences at Universidad
\end{abstract}

FECHA DE RECEPCIÓN: FECHA DE REVISIÓN: APROBACIÓN:
5 de marzo de 2012

29 de abril de 2012

4 de mayo de 2012
PARA CITAR ESTE artículo / to CITE thIS ARTICLE

Montoya, L., Montoya, I. \& Rojas, S. Percepción de la calidad y satisfacción de la elección de los estudiantes de primer semestre en la Facultad de Ciencias Económicas de la Universidad Nacional de Colombia. Poliantea 8 (14), 71-94. 
la Universidad Nacional de Colombia, con la cual se buscó indagar los niveles de percepción de calidad y satisfacción en la elección tanto de la universidad como del programa elegido. Para ello se aplicó una encuesta estructurada, asistida, escrita, aleatoria, a los nuevos estudiantes. El estudio surgió de la necesidad, antes percibida, por parte de la universidad, de conocer la posición de los jóvenes que aspiran a ingresar a ella y reconocer si el factor bajo costo es el indicador más importante para que se quieran vincular los estudiantes. También se indagó sobre aspectos tales como la percepción de calidad de los estudiantes, de los profesores y de los egresados de la facultad, así como la satisfacción que tienen tanto de la universidad como del programa que actualmente se encuentran estudiando. Los resultados son alentadores, demuestran que los estudiantes se encuentran muy satisfechos de su ingreso a la universidad y en general con los programas académicos que adelantan. Estos estudios son de gran relevancia porque permiten encontrar aspectos de mejoramiento y entender que los estudiantes, aunque sean de una universidad pública, deben sentirse satisfechos, y que sus percepciones son importantes.

Palabras clave: percepción, satisfacción, calidad de la educación superior.
Nacional de Colombia. This investigation aimed to know the perception levels about the quality and satisfaction in the selection of the University and the program itself. For this, we applied a structured, assisted, written, and random survey to the new students. This study resulted from the need, perceived firstly from the University, to know the position of the new candidates and to confirm if the low cost factor is the most important indicator for students to desire to study there. We also asked about different aspects, such as the quality perception of students, faculty, and graduates of the Faculty, as well as the level of satisfaction they have regarding the University and the program they are currently studying. The results are encouraging because they show that students are very satisfied with the University and the academic programs they are enrolled in. These studies are relevant because they allow finding aspects to improve and understanding that students, even from a public university, should be satisfied and feel that their perceptions are important.

Keywords: Perception, satisfaction, quality of higher education. 


\title{
Percepción de la calidad y satisfacción de la elección de los estudiantes de primer semestre en la Facultad de Ciencias Económicas de la Universidad Nacional de Colombia
}

\begin{abstract}
Luz Alexandra Montoya R. (Colombia)
Profesora Asociada, Facultad de Ciencias Económicas, coordinadora nacional del Concurso Docente.

Iván Alonso Montoya R. (Colombia)

Profesor Asociado, Facultad de Minas, Universidad Nacional de Colombia, Núcleo Robledo, Medellín - Colombia

Sandra Patricia Rojas (México)

Directora del Departamento de Investigación, Desarrollo e Innovación, Institución Universitaria Politécnico Grancolombiano.
\end{abstract}

\section{Introducción}

La Universidad Nacional de Colombia es la más importante del país. Desde su fundación, en la segunda mitad del siglo XIX, se ha reconocido como una universidad nacional pública y del Estado. Este carácter hace que sea de proyección nacional y se le haya otorgado un papel central en la construcción de la unidad nacional, el estudio y el enriquecimiento del patrimonio cultural, natural y ambiental del país y el análisis de los problemas nacionales y la formulación independiente de soluciones a estos. Por su naturaleza pública esta universidad tiene un carácter pluralista, policlasista, laico y nacional, no responde a intereses particulares, lo cual le permite pensar y proponer soluciones más allá de los intereses de la rentabilidad económica (Montoya et al., 2000). 
La Universidad Nacional tiene una alta demanda nacional, del total de 64847 inscritos para el primer semestre de 2011 solo fueron admitidos 5954 en todas las sedes (Amazonia, Orinoquia, Bogotá, Medellín, Manizales, Palmira, San Andrés); esto hace que únicamente el $8,4 \%$ de los aspirantes pudieron ingresar a ella, lo que denota los índices de calidad y exigencia tanto de la universidad como de los nuevos estudiantes.

Sin embargo, la Universidad Nacional no puede quedarse en las estadísticas, debe propender por una visión integral de sus aspirantes; es importante, tanto para ella como para la Facultad de Ciencias Económicas y para el programa curricular de Administración de Empresas, conocer las expectativas que tienen los estudiantes al ingresar al programa, pues al conocerlas será más fácil para ella satisfacer estas necesidades.

En ese sentido, se ha encontrado que uno de los aspectos en el que los estudiantes se fijan especialmente es el futuro, por ello revisan el mercado laboral cuando de escoger una carrera se trata. Esta decisión, sin duda, marcará su vida (López, 2011) y según los datos de 2011, la carrera más demandada es Administración de Empresas, seguida por Ingeniería
Industrial, Administración de Negocios Internacionales e Ingeniería en Informática y Sistemas.

En este sentido, el rector del Colegio de Estudios Superiores en Administración de Colombia, José Manuel Restrepo, afirmó:

Es cierto que al final del ejercicio, la carrera de Administración seguirá siendo de las más demandadas. Esta disciplina, al ser una profesión, forma gente práctica, y esa visión significa que cuando el administrador llega a la empresa, tiene mayor facilidad para entender los problemas y necesidades de la compañía. Aun así, las empresas buscan más que una disciplina concreta (López, 2011).

Con esto se señala cómo a la hora de elegir un programa de educación la tendencia no se centra en continuar una tradición familiar, una vocación, $o$ adquirir un estatus social, y lo que se busca es estudiar carreras prácticas, que sea de provecho e inmediatez su ejercicio profesional en el país, y que además permita una rápida y exitosa inserción en el competido mercado laboral (López, 2011).

Es por eso que este estudio de las expectativas de los nuevos estudiantes de Administración de Empresas es tenido en cuenta para poder evaluar al final de sus programas si estas 
se cumplieron, así se encuentra que para el programa de Administración de Empresas, sede Bogotá, en el primer semestre de 2011 se presentaron 1937 aspirantes, de los cuales fueron admitidos 90 (4,64\%) (Admisiones, 2011).

\section{Antecedentes de la investigación}

En el año 2000 un grupo de investigadores inició el primer "Estudio de la imagen de la Universidad Nacional", acompañado por el vicerrector general, profesor Gustavo Montañez; el director del proyecto, Roberto Rosero, profesores invitados y la empresa de investigación de mercados Napoleón Franco. El objetivo de dicha investigación fue el de identificar la imagen que tienen los bachilleres (estudiantes de último año) de Bogotá sobre la Universidad Nacional de Colombia, con unos objetivos específicos, tales como los de saber qué carrera están pensando estudiar, cuál es la universidad escogida y en qué lugar se encuentra la Universidad Nacional dentro de estas opciones; determinar cuál sería la carrera y universidad que escogerían si pudieran tener acceso a una beca universitaria, conocer el cambio de posición de la Universidad Nacional, explorar las razones de dicho cambio $y$ esclarecer las razones de escogencia o no de la Universidad Nacional.

La ficha técnica de dicho estudio fue una entrevista personal con cuestionario estructurado, muestreo bietápico estratificado, con selección aleatoria sistemática de las unidades muestrales. Además se seleccionó en todos los cursos un número (n) de alumnos. Grupo objetivo: jóvenes, hombres y mujeres, estudiantes de último año de bachillerato que piensan seguir carrera universitaria. Fecha de realización: junio 11 - julio 27 de 1999. Tamaño de la muestra: 1203 entrevistas sin ponderar (309 de estrato alto, 404 de estrato medio y 400 de estrato bajo). En total, 1210 entrevistas ponderadas por estrato (54 alto, 77 medio, 1079 bajo), en las que participaron los autores del presente documento. Este estudio recogió, además, un análisis previo efectuado en julio de 1986 con 50 estudiantes universitarios de último año de la Universidad de La Sabana, quienes entrevistaron técnica y controladamente a $351 \mathrm{em}$ presas bogotanas, grandes y medianas. Fueron abordados los gerentes de mayor nivel y los de selección de personal. La encuesta hecha buscaba determinar el centro docente preferido para el suministro de recién egresados como administradores de 
empresas. A modo de conclusiones primordiales de dicho estudio puede señalarse que, para el año 2000, la carrera preferida en el estrato alto era Ingeniería Industrial, en tanto que la de Sistemas se prefería en el medio y en el bajo (v. tabla 1).

Tabla No. 1. Carreras preferidas en el 2000

\begin{tabular}{|l|c|c|c|}
\hline \multicolumn{1}{|c|}{ Carrera } & Alto & Medio & Bajo \\
\hline Ing. Industrial & 11 & $\%$ & $\%$ \\
\hline Medicina & 9 & 8 & 8 \\
\hline Bellas Artes & 6 & 2 & 4 \\
\hline $\begin{array}{l}\text { Administración de } \\
\text { Empresas }\end{array}$ & 5 & 3 & 5 \\
\hline Ing. Electrónica & 4 & 3 & 4 \\
\hline Otras de la Salud & 3 & 7 & 7 \\
\hline Ing. de Sistemas & 2 & 11 & 13 \\
\hline $\begin{array}{l}\text { Ciencias de la } \\
\text { Educación }\end{array}$ & 2 & 6 & 9 \\
\hline
\end{tabular}

Fuente: Montoya et al. (2000)

Como principales razones para estudiar en la Universidad Nacional de Colombia se señaló la económica, con el (49\%), su prestigio (25\%) y su calidad (24\%), frente a otras universidades colombianas (v. tabla 2).
Tabla No. 2. Razones por las cuales eligió estudiar en la universidad

\begin{tabular}{|c|c|c|c|c|}
\hline Razones & $\begin{array}{l}\text { 흠 } \\
\text { 음 }\end{array}$ & 름 & 蒫 & 혼 \\
\hline & $\%$ & $\%$ & $\%$ & $\%$ \\
\hline Es económica/barata & 49 & 1 & & 53 \\
\hline Es una buena universidad & 25 & 33 & 16 & 24 \\
\hline $\begin{array}{l}\text { Por su buen nivel } \\
\text { académico }\end{array}$ & 24 & 37 & 17 & 18 \\
\hline Buenas referencias & 13 & 12 & 5 & 13 \\
\hline El gusto de estudiar allí & 7 & 2 & 12 & 5 \\
\hline Tiene prestigio & 7 & 7 & 16 & 5 \\
\hline $\begin{array}{l}\text { Es la que mejor dicta la } \\
\text { carrera }\end{array}$ & 6 & 11 & 19 & 13 \\
\hline $\begin{array}{l}\text { La única que enseña esa } \\
\text { carrera }\end{array}$ & 3 & 10 & & 11 \\
\hline
\end{tabular}

Fuente: Montoya et al. (2000).

Esto se corrobora con los atributos que se le dieron a la Universidad Nacional en cuanto a la percepción de calidad, exigencia y reconocimiento (v. tabla 3 ).

Tabla No. 3. Atributos según la universidad

\begin{tabular}{|l|c|c|c|}
\hline \multicolumn{1}{|c|}{ Atributos } & Nacional & Javeriana & Andes \\
\hline $\begin{array}{l}\text { El nivel y la } \\
\text { exigencia } \\
\text { académica son } \\
\text { altos }\end{array}$ & 76 & 61 & 57 \\
\hline $\begin{array}{l}\text { La calidad de } \\
\text { la educación } \\
\text { es alta }\end{array}$ & 74 & 60 & 57 \\
\hline $\begin{array}{l}\text { La calidad de } \\
\text { sus docentes } \\
\text { es alta }\end{array}$ & 69 & 56 & 55 \\
\hline $\begin{array}{l}\text { Las facilidades } \\
\text { para el pago } \\
\text { de la matrícula } \\
\text { son altas }\end{array}$ & 69 & 24 & 22 \\
\hline $\begin{array}{l}\text { La calidad de } \\
\text { sus egresados }\end{array}$ & 67 & 54 & 49 \\
\hline
\end{tabular}

sigue... 


...Continuación
\begin{tabular}{|l|c|c|c|}
\hline \multicolumn{1}{|c|}{ Atributos } & Nacional & Javeriana & Andes \\
\hline $\begin{array}{l}\text { El reconoci- } \\
\text { miento de los } \\
\text { egresados }\end{array}$ & 65 & 47 & 49 \\
\hline $\begin{array}{l}\text { La vinculación } \\
\text { de sus } \\
\text { egresados }\end{array}$ & 59 & 52 & 51 \\
\hline $\begin{array}{l}\text { El valor de } \\
\text { la matrícula } \\
\text { en cada } \\
\text { universidad } \\
\text { es alto }\end{array}$ & 48 & 44 & 50 \\
\hline
\end{tabular}

Fuente: Montoya et al. (2000).

Otras conclusiones importantes del estudio señalaron que el $51 \%$ de los estudiantes de Bogotá deseaban estudiar en la Universidad Nacional de Colombia, dada una percepción muy alta de calidad, exigencia y desempeño (Montoya et al., 2000).

\section{Marco conceptual}

El proceso de mercadeo busca conocer las necesidades y los deseos de las personas que deben satisfacerse; sin embargo, este proceso no es sencillo. Los consumidores no son racionales al momento de decidir y existen muchas influencias que pueden afectar el proceso de compra y consumo. La educación, vista como una necesidad, debe proporcionar satisfacción, no solo en la obtención de un título, sino en el proceso de formación.

Para entender estos procesos en los nuevos estudiantes, una de las metodologías que debe seguirse requiere fundamentarse en la percepción. Estos estudios han considerado dos factores influyentes: la cultura y la posición económica. El primer factor considera a la cultura sobre la percepción. Los valores y creencias del sujeto están orientados por las prácticas culturales específicas de cada grupo. Se señala a la cultura como un factor que influye bastante en la conducta del sujeto debido a que provee identidad a los miembros de la estructura social. La identidad es un proceso que afecta la emoción y la cognición, $\mathrm{y}$, por consiguiente, la percepción (Cole, 2003 \& Velazco, 2011). Rosenthal y Rubin (1978) iniciaron los estudios de la influencia que tienen las expectativas de unas personas sobre otras. Burón (1995) describió el proceso por medio del cual las expectativas de unos influyen en otros, y señaló tres partes fundamentales: formación de expectativas, establecimiento de la realización de estas y confirmación. Una aplicación de dicho fenómeno en la educación la proporcionan Pichardo y otros (2007):

1) Se forman las expectativas. Como ejemplo pueden señalarse dos expectativas que ocurren en el ámbito universitario, 
una se refiere a la influencia del profesorado en el alumnado: "es imposible aprobar con el profesor de tal asignatura"; y otra en una situación inversa: "con los cursos de tal titulación es imposible dar clases".

2) Se establecen los medios para que las expectativas se realicen. Por ejemplo, no poner todos los recursos disponibles o implicarse menos, tanto en la programación y el diseño de la asignatura por parte del profesorado, como en la planificación y el estudio por parte del alumnado. Esto puede ser porque se percibe que la probabilidad de enseñar o aprender se considera mínima.

3) Se describe la confirmación de las expectativas. Al no esforzarse igual o más que en otras ocasiones provoca resultados negativos y la profecía se cumple; así las expectativas se afianzan más (Pichardo et al., 2007, p. 20).

Prakash (1984) señala cómo las expectativas de una persona se relacionan directamente con la satisfacción de los clientes o consumidores. Zeithaml, Parasuraman y Berry $(1990,1993)$ proponen un modelo conceptual de las expectativas del servicio del cliente, con el cual se demuestra la distinción entre la satisfacción de este y la evaluación de la calidad del servicio. Las conclusiones de todos estos trabajos consisten, entre otras, en que las expectativas están relacionadas con la satisfacción posterior en la calidad del servicio, y que el estudio y la consideración de las expectativas de los clientes ayudan a garantizar dicha calidad (Pichardo, 2007; Casero, 2010); las percepciones de calidad de servicio reflejan las apreciaciones del consumidor en un momento específico de tiempo. Por el contrario, los juicios de satisfacción del consumidor tienen su origen en la experiencia, lo cual es reflejo final de un proceso lleno de elementos emocionales y cognitivos (Oliver, 1981; Rust \& Oliver, 1994 Caetano, 2003).

El concepto de expectativa señala que las personas hacen determinadas exigencias sobre servicios con base en sus normas, valores, deseos y necesidades personales, lo cual hace que este proceso sea bastante individualista. Por esta razón las expectativas serán siempre específicas y pueden ser alteradas en el transcurso del tiempo, durante nuevas situaciones. Por otro lado, las expectativas son determinadas, 
no solo por los individuos aisladamente, sino también por grupos de referencia, ambientes externos, valores éticos, tiempos, así como por el proveedor del servicio. Las expectativas se definen, básicamente, como los deseos de los consumidores (Parasuraman, Berry \& Zeithaml, 1988; Caetano, 2003; Cronin \& TayIor, 1994).
La percepción del buen servicio se da en relación con la calidad esperada y la calidad experimentada, que proporcionan en total la calidad percibida. La calidad esperada se relaciona mediante la expectativa que se tenga de ella y la experimentada con una experiencia real con el producto o servicio, lo cual puede verse en la figura 1.

Figura No. 1. Criterios de buen servicio

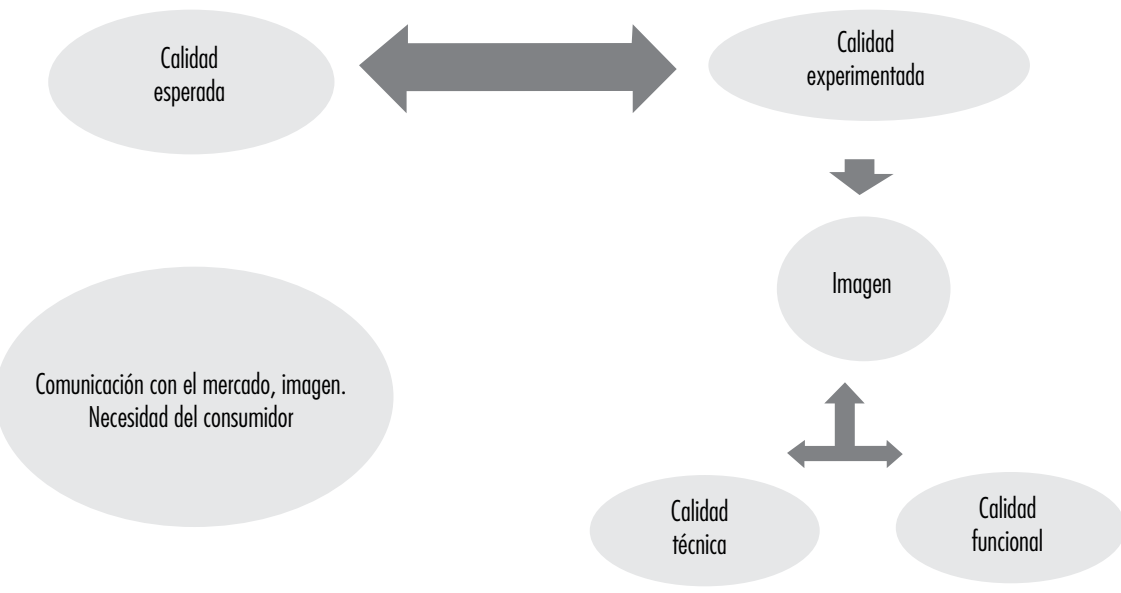

Fuente: Gronross, 1998.

La satisfacción es la evalua- expectativas. Morales (2003) enconción que realiza el cliente respec- tró múltiples definiciones de la sato de un servicio y depende de que tisfacción, las cuales resume en la este responda a sus necesidades y tabla 4: 
Tabla No. 4. Revisión del constructo de satisfacción en los usuarios o consumidores

\begin{tabular}{|c|c|c|c|c|}
\hline Autores & Definición & Criterio & Objeto & Fase \\
\hline Howard y Sheth (1969) & $\begin{array}{l}\text { Estado cognitivo de la } \\
\text { adecuación o inadecuación } \\
\text { de la recompensa recibida } \\
\text { con respecto a la inversión } \\
\text { realizada. }\end{array}$ & Estado cognitivo & $\begin{array}{l}\text { Adecuación o } \\
\text { inadecuación de la } \\
\text { recompensa a la inversión }\end{array}$ & Después del consumo \\
\hline Hunt (1977) & $\begin{array}{l}\text { Evaluación que analiza si una } \\
\text { experiencia de consumo es } \\
\text { al menos tan buena como se } \\
\text { esperaba. }\end{array}$ & $\begin{array}{l}\text { Evaluación de una } \\
\text { experiencia }\end{array}$ & $\begin{array}{l}\text { Analizar si la experiencia } \\
\text { alcanza o supera las } \\
\text { expectativas }\end{array}$ & Durante el consumo \\
\hline Oliver (1980-1981) & $\begin{array}{l}\text { Estado psicológico final } \\
\text { resultante cuando la } \\
\text { sensación que rodea a } \\
\text { la discrepancia de las } \\
\text { expectativas se une con } \\
\text { los sentimientos previos } \\
\text { acerca de la experiencia de } \\
\text { consumo. }\end{array}$ & $\begin{array}{l}\text { Evaluación, estado } \\
\text { psicológico final y } \\
\text { respuesta emocional } \\
\text { dada por estándar } \\
\text { inicial en cuanto a las } \\
\text { expectativas }\end{array}$ & $\begin{array}{l}\text { Discrepancia entre } \\
\text { las expectativas y el } \\
\text { rendimiento percibido } \\
\text { del producto consumido. } \\
\text { Evaluación de la sorpresa } \\
\text { inherente a la experiencia } \\
\text { de compra } 0 \text { adquisición }\end{array}$ & $\begin{array}{l}\text { Durante la compra o el } \\
\text { consumo }\end{array}$ \\
\hline $\begin{array}{l}\text { Churchill y Surprenant } \\
\text { (1982) }\end{array}$ & $\begin{array}{l}\text { Respuesta a la compra } \\
\text { de productos o uso de } \\
\text { servicios que se deriva } \\
\text { de la comparación por } \\
\text { el consumidor, de las } \\
\text { recompensas y sus costos de } \\
\text { compra con relación a sus } \\
\text { consecuencias esperadas. }\end{array}$ & Resultado, actitud & $\begin{array}{l}\text { Comparación de los } \\
\text { costos y las recompensas } \\
\text { con las consecuencias } \\
\text { esperadas de la compra }\end{array}$ & $\begin{array}{l}\text { Después de la compra } 0 \\
\text { del consumo }\end{array}$ \\
\hline $\begin{array}{l}\text { Swan, Trawick y Carroll } \\
\text { (1982) }\end{array}$ & $\begin{array}{l}\text { Juicio evaluativo o cognitivo } \\
\text { que analiza si el producto } \\
\text { presenta un resultado bueno } \\
\text { o pobre o si el producto es } \\
\text { sustituible o insustituible. } \\
\text { Respuestas afectivas hacia el } \\
\text { producto. }\end{array}$ & $\begin{array}{l}\text { Juicio o evaluación } \\
\text { global, determinado } \\
\text { por respuestas } \\
\text { afectivas y cognitivas }\end{array}$ & Resultados del producto & $\begin{array}{l}\text { Durante o después del } \\
\text { consumo }\end{array}$ \\
\hline $\begin{array}{l}\text { Westbrock y Reilly } \\
\text { (1983) }\end{array}$ & $\begin{array}{l}\text { Respuesta emocional causada } \\
\text { por un proceso evaluativo- } \\
\text { cognitivo en el que las } \\
\text { percepciones son un objeto, } \\
\text { acción o condición y se } \\
\text { comparan con necesidades y } \\
\text { deseos del individuo. }\end{array}$ & Respuesta emocional & & Después de la compra \\
\hline $\begin{array}{l}\text { Cadotte, Woodruffy y } \\
\text { Jenkins (1987) }\end{array}$ & $\begin{array}{l}\text { Sensación desarrollada a } \\
\text { partir de la evaluación de una } \\
\text { experiencia de uso. }\end{array}$ & $\begin{array}{l}\text { Sensación causada por } \\
\text { la evaluación }\end{array}$ & Experiencia de uso & Después del consumo \\
\hline $\begin{array}{l}\text { Tse, Nicosia y Wilton } \\
\text { (1990) }\end{array}$ & $\begin{array}{l}\text { Respuesta del consumidor } \\
\text { a la evaluación de la } \\
\text { discrepancia percibida entre } \\
\text { expectativas y el resultado } \\
\text { final percibido en el producto } \\
\text { tras su consumo. Proceso } \\
\text { multidimensional y dinámico. }\end{array}$ & $\begin{array}{l}\text { Interactúan } \\
\text { actividades mentales } \\
\text { y conductuales a lo } \\
\text { largo del tiempo. } \\
\text { Respuesta causada por } \\
\text { la evaluación }\end{array}$ & $\begin{array}{l}\text { Discrepancia percibida } \\
\text { entre expectativas (otras } \\
\text { normas de resultado) } \\
\text { y el resultado real del } \\
\text { producto }\end{array}$ & Después del consumo \\
\hline
\end{tabular}


...Continuación

\begin{tabular}{|c|c|c|c|c|}
\hline Autores & Definición & Criterio & Objeto & Fase \\
\hline $\begin{array}{l}\text { Westbrook y Oliver } \\
\text { (1991) }\end{array}$ & $\begin{array}{l}\text { Juicio evaluativo posterior a } \\
\text { la selección de una compra } \\
\text { específica }\end{array}$ & Juicio evaluativo & $\begin{array}{l}\text { Selección de compra } \\
\text { especifica }\end{array}$ & Posterior a la selección \\
\hline Mano y Oliver (1993) & $\begin{array}{l}\text { Respuesta del consumidor } \\
\text { asociada a la compra } \\
\text { del producto o servicio } \\
\text { consumido. }\end{array}$ & $\begin{array}{l}\text { Respuesta cognitiva y } \\
\text { afectiva }\end{array}$ & $\begin{array}{l}\text { Respuesta promovida } \\
\text { por factores cognitivos y } \\
\text { afectivos }\end{array}$ & Posterior al consumo \\
\hline $\begin{array}{l}\text { Halstead, Hartman y } \\
\text { Schmidt (1994) }\end{array}$ & $\begin{array}{l}\text { Respuesta afectiva asociada } \\
\text { a una transacción específica } \\
\text { resultante de la comparación } \\
\text { del resultado del producto } \\
\text { con algún estándar fijado con } \\
\text { anterioridad a la compra. }\end{array}$ & Respuesta afectiva & $\begin{array}{l}\text { Resultado del producto } \\
\text { comprado con un } \\
\text { estándar anterior a la } \\
\text { compra }\end{array}$ & $\begin{array}{l}\text { Durante o después del } \\
\text { consumo }\end{array}$ \\
\hline Oliver (1996) & $\begin{array}{l}\text { Juicio del resultado que un } \\
\text { producto o servicio se ofrece } \\
\text { para un nivel suficiente de } \\
\text { realización en el consumo. }\end{array}$ & $\begin{array}{l}\text { Respuesta evaluativa } \\
\text { del nivel de realización }\end{array}$ & Producto o servicio & Durante el consumo \\
\hline
\end{tabular}

Fuente: Morales (2003).

En general se puede señalar que la satisfacción implica:

- La existencia de un objetivo que el consumidor desea alcanzar.

- La consecución de este objetivo solo puede ser juzgada tomando como referencia un estándar de comparación.

- El proceso de evaluación de la satisfacción implica como mínimo la intervención de dos estímulos: un resultado y una referencia o estándar de comparación (Oliver, 1993, 1989).

Desde la perspectiva del cliente, la dimensión de la calidad es la subjetiva. Los clientes se forjan una expectativa de calidad que procede de tres fuentes (Zeithaml, 1988): 1) necesidades personales: esperan encontrar un producto que los satisfaga; 2) expectativas: las características y experiencias de los servicios que transmiten terceras personas crean expectativas de calidad de los servicios, en restaurantes, hoteles, compañías aéreas, peluquerías, entidades financieras, servicios profesionales; los consumidores emplean esta información como punto de referencia para hacer comparaciones entre lo esperado y lo recibido; 3) experiencia: los compradores de servicios pueden tener experiencias como tales; las adquisiciones continuadas les permiten fijarse un nivel de calidad que emplean como punto de referencia; en cuanto el servicio se aleje de ese nivel de calidad habitual, se apreciarán diferencias de ella. 
Vrontis et al. (2007) propusieron un modelo de elección de estudiantes en la educación superior contemporánea en el cual señalan que los elementos que se conjugan en la decisión de un estudiante son de diversos grados:

1) Las características de los estudiantes, en términos sociales, demográficos y económicos, tales como raza, ingresos, estrato social, educación de los padres, cultura familiar, personalidad de sus padres, organización familiar, religión y sexo, entre otras.

2) Características de la institución de educación superior: composición social, calidad, currículo, programas.

3) Influencias y medios de comunicación: padres, consejeros, amigos, medios de comunicación masivos, entre otros.

4) Atributos personales: posición entre sus compañeros de clase, habilidades y aptitudes académicas, desempeño, logros, autoimagen, personalidad, estilo de vida, aspiraciones educacionales.
5) Ambiente: estructura del empleo, condiciones económicas y culturales.

6) Características del colegio: precio/costo, ayudas, becas, financiamiento, tamaño, disponibilidad de los programas, localización, tipo de institución (pública o privada).

7) Acciones de la institución educativa: actividades de posicionamiento, políticas académicas y de admisión, ayudas, información, campus, admisiones.

El proceso de elección del estudiante se encuentra influenciado por cada uno de ellos; su primer nivel son las aspiraciones universitarias (Astin, 1993; Biscarri, Filelia, Jove, 2008), en el que inicia una etapa de búsqueda, recolecta información y envía aplicaciones para vincularse a una institución (López, 2004; Palomero, 2004).

El modelo de Vrontis et al. (2007) incluye estos determinantes en un medio global y cultural (v. figura 2) que conduce a ver la educación como un negocio que debe tener en cuenta aspectos de mercadeo. 
Figura No. 2. Modelo de elección de educación superior para países en desarrollo

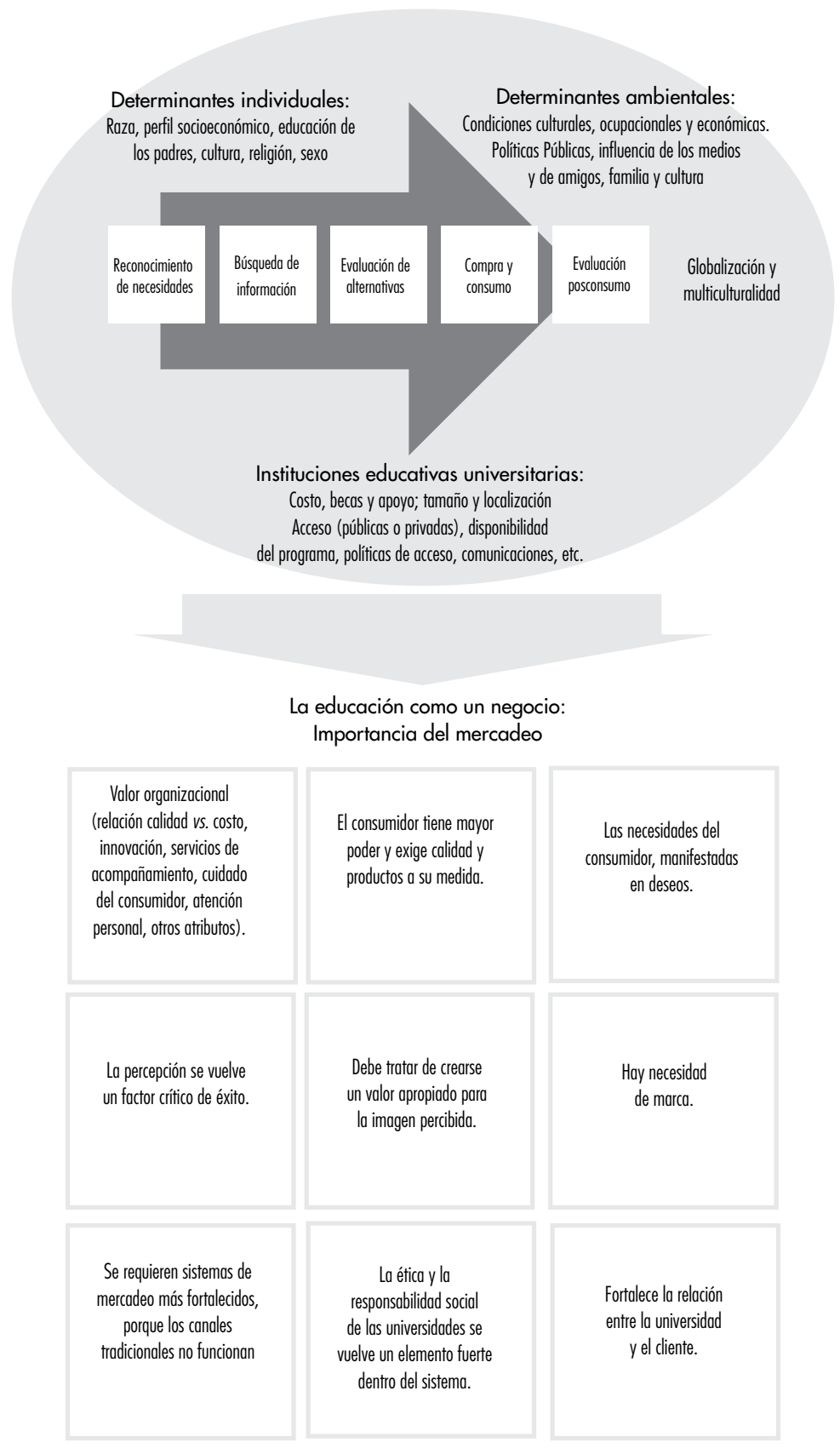

Fuente: Adaptado de Vrontis (2007). 


\section{Metodología del estudio}

Como premisa, la Universidad Nacional de Colombia se ha caracterizado por ofrecer a sus estudiantes las herramientas necesarias para lograr el desarrollo de sus capacidades intelectuales y de sus intereses personales en distintos ámbitos de las ciencias y las artes; de tal modo, se hace necesario realizar sondeos de opinión que vislumbren la percepción que los estudiantes tienen tanto de su universidad como de sus programas académicos.

Es por ello que se realizó una encuesta estructurada asistida a 86 de los 304 admitidos para el primer semestre de 2011 en la Facultad de Ciencias Económicas (www. admisiones.unal.edu.co), 28,28\% de la población. Esta muestra tiene un error de $9,9 \%$, un nivel de confianza de $97 \%$ (fórmula para población finita), y su objetivo fue el de obtener información acerca de su expectativa con respecto a sus carreras y a la Universidad Nacional en sí.

\section{Descripción de las variables}

Para el análisis de las percepciones acerca de la calidad y la satisfacción con la elección de su carrera, fue adoptado el modelo de Vrontis (2007), en el cual se tuvieron en cuenta las variables que se presentan en la tabla 5.

Tabla No. 5. Variables del estudio

\begin{tabular}{|c|c|}
\hline Variable & Descripción \\
\hline $\begin{array}{l}\text { Caracterización de los } \\
\text { estudiantes }\end{array}$ & $\begin{array}{l}\text { Segmentación demográfica, } \\
\text { edad, género, estrato social, } \\
\text { lugar del hogar. }\end{array}$ \\
\hline $\begin{array}{l}\text { Caracterización de la } \\
\text { universidad }\end{array}$ & $\begin{array}{l}\text { Motivaciones de la selección } \\
\text { de la universidad y de la } \\
\text { carrera. }\end{array}$ \\
\hline $\begin{array}{l}\text { Influencias en la decisión y } \\
\text { medios de transmisión de la } \\
\text { información }\end{array}$ & $\begin{array}{l}\text { Quiénes influyeron en la } \\
\text { decisión tanto de la selección } \\
\text { de la universidad como del } \\
\text { programa. }\end{array}$ \\
\hline Atributos personales & $\begin{array}{l}\text { Tales como motivaciones de } \\
\text { estudio tanto en la universidad } \\
\text { como en la carrera. }\end{array}$ \\
\hline Ambiente & $\begin{array}{l}\text { Condiciones del estudiante de } \\
\text { primer semestre. }\end{array}$ \\
\hline Características de la institución & $\begin{array}{l}\text { Percepción de calidad de la } \\
\text { institución. }\end{array}$ \\
\hline $\begin{array}{l}\text { Percepción de estudiantes, } \\
\text { egresados y profesores }\end{array}$ & $\begin{array}{l}\text { Características de los } \\
\text { estudiantes, profesores y } \\
\text { egresados de la institución. }\end{array}$ \\
\hline Acciones de la institución & $\begin{array}{l}\text { Como la de darle al estudiante } \\
\text { la posibilidad de una beca. }\end{array}$ \\
\hline Satisfacción general & $\begin{array}{l}\text { Nivel de satisfacción general } \\
\text { frente a la posibilidad de tener } \\
\text { una beca. }\end{array}$ \\
\hline
\end{tabular}

Fuente: Adaptado de Vrontis (2007).

De acuerdo con dichas variables se desarrolló un cuestionario autoaplicado, el cual desarrollaba las variables en preguntas de acuerdo con la escala de Likert. El cuestionario empleado para el estudio constó de dieciséis preguntas, en las que se pidió a los nuevos estudiantes de la facultad calificar sus respuestas en una escala de 1 a 5 . 


\section{Resultados}

En la caracterización de la muestra se pudo observar que más del $90 \%$ de los encuestados son menores de 20 años, también parece relevante resaltar que el 78\% de los entrevistados están entre los 16 años y los 18 años. En cuanto al sexo se tiene que un $64 \%$ de los entrevistados son hombres y un 36\% mujeres. Predominan los estudiantes de estratos socioeconómicos 2, 3 y 4 (bajo, medio y medio alto); el $40 \%$ de los entrevistados son de estrato bajo.

En cuanto a la carrera que estudian, la muestra, a pesar de ser aleatoria, resultó equitativa en este aspecto, pues se tiene un $36 \%$ de estudiantes en Administración de Empresas, un $34 \%$ en Contaduría Pública y un 30\% en Economía.

Con respecto a la localidad donde viven, se puede ver que los estudiantes proceden de casi todas las localidades de Bogotá y un 13\% de ellos viven fuera de la ciudad, en municipios como Zipaquirá, Facatativá, Cogua, Sopó y Mosquera (pequeñas ciudades cercanas a la ciudad de Bogotá, pero cuyo recorrido puede requerir de hasta dos horas en servicio público).
De esta forma se tiene que la muestra poblacional entrevistada la conforman, en su mayoría, hombres jóvenes, de estratos medios, que viven en barrios populares o fuera de la ciudad y que estudian primer semestre en cualquiera de las tres carreras de pregrado en la Facultad de Ciencias Económicas de la Universidad Nacional de Colombia.

Acto seguido, en la encuesta se busca saber si los estudiantes creen haber hecho una elección correcta al estudiar en la Universidad Nacional; los resultados obtenidos muestran que el $82 \%$ opina afirmativamente, mientras que el $18 \%$ restante no lo juzga así o no respondió la pregunta, lo que muestra un nivel de conformidad bastante alto tanto con la universidad como con sus respectivas carreras.

En cuanto a las motivaciones que tuvieron para elegir a la Universidad Nacional se tiene, según los resultados, que la principal motivación fue el nivel académico; en segundo lugar, la calidad de los docentes; y tercero, el prestigio y la imagen de la universidad (v. tabla 6). 
Tabla No. 6. Motivaciones para estudiar en la Universidad Nacional

\begin{tabular}{|c|c|c|c|c|c|c|c|}
\hline Motivaciones & Muy Alta & Alta & Intermedia & Poca & Muy poca & Ninguna & $N / R$ \\
\hline Costo & 41 & 22 & 11 & 4 & 3 & 4 & 1 \\
\hline $\begin{array}{l}\text { Prestigio e } \\
\text { imagen }\end{array}$ & 65 & 13 & 8 & 0 & 0 & 0 & 0 \\
\hline Nivel académico & 72 & 14 & 0 & 0 & 0 & 0 & 0 \\
\hline $\begin{array}{l}\text { Calidad de los } \\
\text { docentes }\end{array}$ & 51 & 30 & 4 & 1 & 0 & 0 & 0 \\
\hline $\begin{array}{l}\text { Calidad del } \\
\text { programa }\end{array}$ & 41 & 33 & 9 & 1 & 0 & 1 & 1 \\
\hline $\begin{array}{l}\text { Es la que mejor } \\
\text { enseña la carrera }\end{array}$ & 24 & 30 & 19 & 6 & 0 & 6 & 1 \\
\hline $\begin{array}{l}\text { Es la única que } \\
\text { dicta la carrera }\end{array}$ & 1 & 3 & 3 & 6 & 10 & 61 & 2 \\
\hline $\begin{array}{l}\text { Buenas } \\
\text { referencias }\end{array}$ & 40 & 32 & 9 & 3 & 0 & 1 & 1 \\
\hline $\begin{array}{l}\text { Cercanía de la } \\
\text { casa }\end{array}$ & 2 & 8 & 6 & 11 & 11 & 48 & 0 \\
\hline $\begin{array}{l}\text { Sugerencia de la } \\
\text { familia }\end{array}$ & 14 & 18 & 17 & 5 & 11 & 20 & 1 \\
\hline
\end{tabular}

Fuente: La investigación.

Otro aspecto estudiado fue el de las motivaciones para elegir la carrera, dentro de las cuales se destacan el gusto por el tema y el desempeño profesional; en esta parte también es de destacar que la sugerencia familiar tuvo muy baja calificación.

Tabla No. 7. Motivaciones para estudiar una carrera en la Universidad Nacional

\begin{tabular}{|l|c|c|c|c|c|c|c|}
\hline Motivaciones & Muy alta & Alta & Intermedia & Poca & Muy poca & Ninguna & N/R \\
\hline $\begin{array}{l}\text { Calidad del } \\
\text { programa }\end{array}$ & 24 & 36 & 14 & 5 & 1 & 5 & 1 \\
\hline $\begin{array}{l}\text { Calidad de los } \\
\text { docentes }\end{array}$ & 31 & 32 & 11 & 5 & 0 & 5 & 2 \\
\hline $\begin{array}{l}\text { Contenidos del } \\
\text { programa }\end{array}$ & 29 & 33 & 15 & 3 & 1 & 4 & 1 \\
\hline $\begin{array}{l}\text { Por sugerencia de } \\
\text { la familia }\end{array}$ & 7 & 13 & 9 & 16 & 13 & 26 & 2 \\
\hline Gusto por el tema & 53 & 20 & 8 & 3 & 1 & 0 & 1 \\
\hline $\begin{array}{l}\text { Desempeño } \\
\text { profesional }\end{array}$ & 46 & 23 & 12 & 1 & 2 & 1 & 1 \\
\hline Salarios & 26 & 22 & 18 & 9 & 3 & 6 & 2 \\
\hline $\begin{array}{l}\text { Querer ser } \\
\text { empresario }\end{array}$ & 31 & 21 & 15 & 3 & 9 & 5 & 2 \\
\hline $\begin{array}{l}\text { Prestigio de los } \\
\text { egresados }\end{array}$ & 35 & 17 & 13 & 7 & 5 & 8 & 1 \\
\hline
\end{tabular}

Fuente: La investigación. 
Con respecto a la inquietud de quiénes fueron los motivadores para elegir la Universidad Nacional de Colombia, los amigos y el colegio parecen ser los principales, y un tanto en contradicción con las respuestas anteriores, los padres o algún otro familiar.

Tras conocer las principales motivaciones, se buscó determinar cómo perciben los estudiantes entrevistados a la Universidad Nacional de Colombia con respecto a sus ventajas y desventajas. En cuanto a las ventajas se tiene, en primer lugar, la calidad de la universidad; en segundo lugar, el prestigio de esta, seguido del de los profesores, y el costo, que también es relevante; los deportes y la flexibilidad no son vistos como ventajas. Entre las desventajas encabezan la lista los paros y los desórdenes; un poco más alejada se cuenta la ubicación, sin que tenga mucha relevancia.

Tabla No. 8. Ventajas de estudiar en la Universidad Nacional de Colombia

\begin{tabular}{|c|c|c|c|c|c|c|c|}
\hline Ventajas & Muy alta & Alta & Intermedia & Poca & Muy Poca & Ninguna & $N / R$ \\
\hline Costo & 56 & 17 & 7 & 1 & 2 & 2 & 1 \\
\hline Calidad & 75 & 10 & 0 & 0 & 0 & 0 & 1 \\
\hline Compañeros & 26 & 28 & 19 & 8 & 1 & 3 & 1 \\
\hline Infraestructura & 30 & 27 & 21 & 6 & 0 & 1 & 1 \\
\hline $\begin{array}{l}\text { Actividades } \\
\text { académicas }\end{array}$ & 35 & 32 & 14 & 1 & 1 & 2 & 1 \\
\hline Deportes & 19 & 22 & 21 & 10 & 3 & 10 & 1 \\
\hline Flexibilidad & 16 & 26 & 22 & 7 & 5 & 9 & 1 \\
\hline Arte y cultura & 36 & 28 & 15 & 4 & 0 & 2 & 1 \\
\hline Profesores & 49 & 26 & 8 & 2 & 0 & 0 & 1 \\
\hline $\begin{array}{l}\text { Programas de } \\
\text { asignaturas }\end{array}$ & 35 & 37 & 11 & 1 & 0 & 1 & 1 \\
\hline $\begin{array}{l}\text { Actividades } \\
\text { culturales }\end{array}$ & 33 & 22 & 18 & 7 & 2 & 3 & 1 \\
\hline Prestigio & 70 & 12 & 2 & 1 & 0 & 0 & 1 \\
\hline
\end{tabular}

Fuente: La investigación.

En lo que respecta a las mate- también fue sobresaliente, destacánrias, calificaron muy bien la calidad dose el conocimiento. La materia de estas, así como los contenidos. En favorita fue Fundamentos de Econocuanto a los profesores, la calificación mía, con un $41 \%$ de predilección. 
Luego se buscó obtener información acerca de cómo se perciben los estudiantes y cómo perciben a sus profesores y a los egresados, teniendo en cuenta ocho aspectos (inteligencia, responsabilidad, amabilidad, buena remuneración, excelentes conocimientos, valoración en las empresas, reconocimiento en la sociedad, valoración en el extranje- ro). Los entrevistados consideran que el estudiante de la Universidad Nacional es inteligente y con excelentes conocimientos; al profesor lo perciben de la misma forma y cabe añadirle el reconocimiento de la sociedad; en cuanto al egresado, también existe la idea de creerlo inteligente, con excelentes conocimientos y reconocido por la sociedad.

Figura No. 3. Aspectos del estudiante de la Universidad Nacional

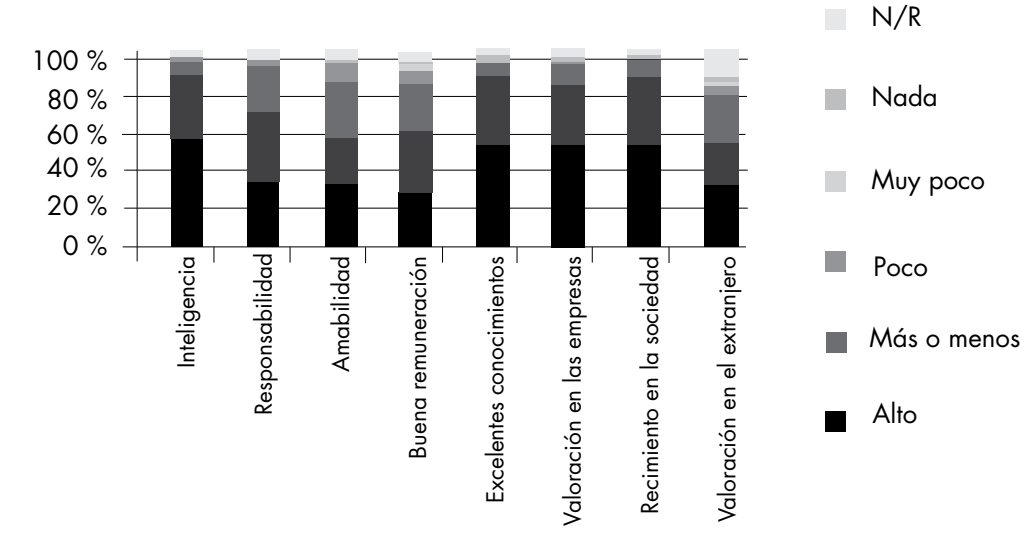

Fuente: La investigación.

Figura No. 4. Aspectos del profesor de la Universidad Nacional

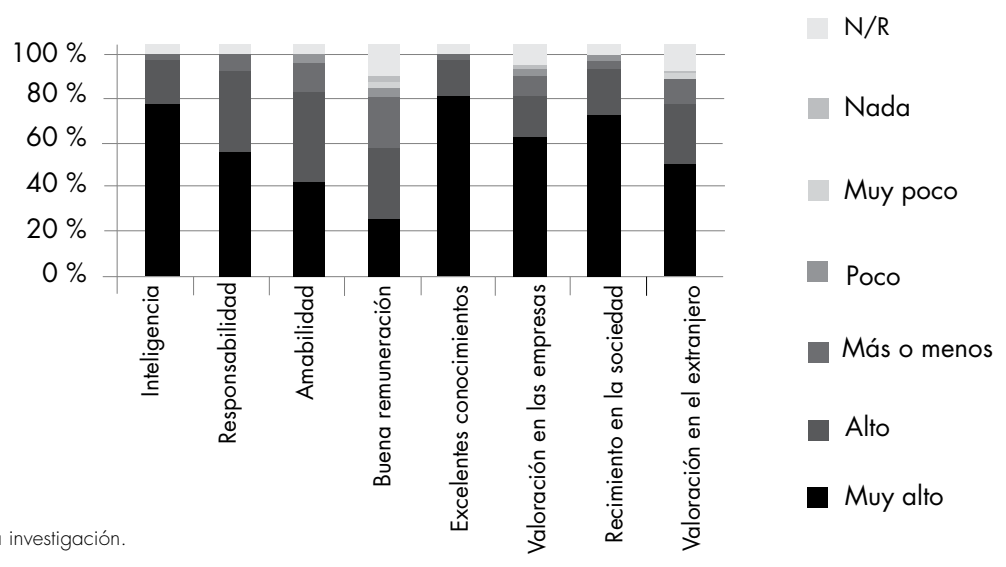


Figura No. 5. Aspectos del egresado de la Universidad Nacional

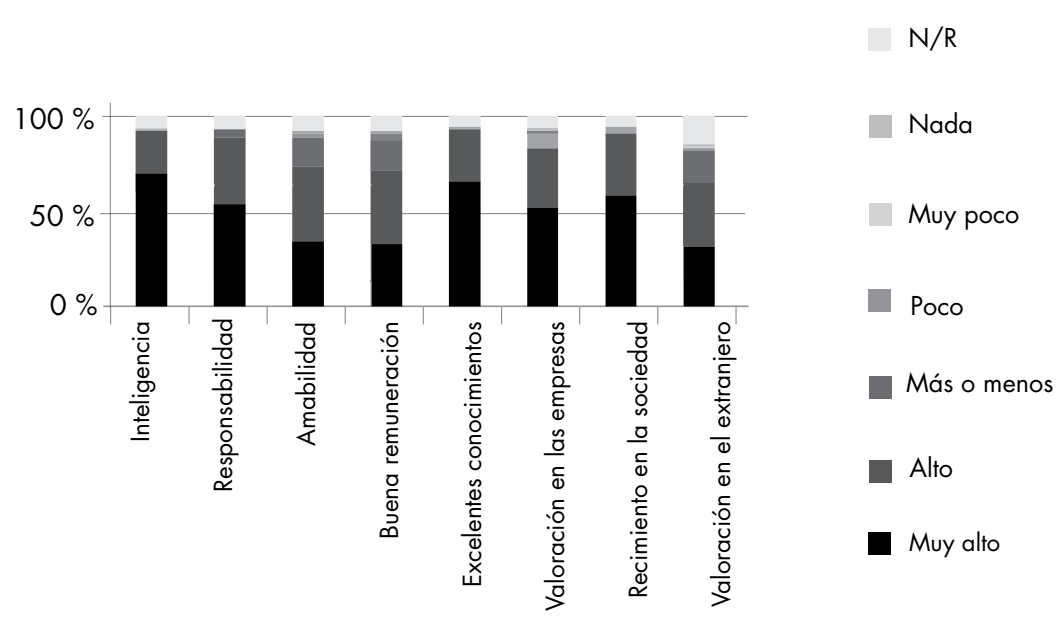

Fuente: La investigación.

Este trabajo además buscó entender el grado de satisfacción de estudiar en la Universidad Nacional, por ello se preguntó a los estudiantes sobre la posibilidad de cambiar de universidad, si tuvieran la oportunidad, a otra nacional o extranjera. Un 58\% señaló que no lo harían, y de contemplar esa posibilidad lo harían de preferencia a una universidad del exterior, entre las que se destacan la Universidad Autónoma de México (UNAM) y la Universidad de Tokio. Este dato es muy importante, porque se les proporciona a los estudiantes la posibilidad de eliminar la dificultad económica. Que el 58\% decida mantenerse en la Universidad Nacional significa que sus motivacio- nes no están restringidas por el bajo costo de esta.

Figura No. 6. Cambio de universidad si pudieran elegir otra

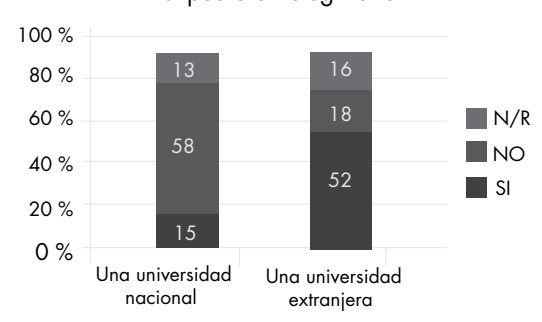

Fuente: La investigación.

Para caracterizar futuras dificultades que produjeran deserción de sus programas, se le preguntó a los estudiantes cuáles motivos impedirían seguir sus carreras. Comenzando por el hecho de que el desempeño de los egresados en el campo laboral es alto, 
seguido de la alta calidad de la educación, lo importante de esta pregunta es que se ratifican las respuestas del inicio de la encuesta en cuanto a que los evaluados creen haber elegido bien la universidad y su programa académico. Sin embargo, es preocupante que los estudiantes, en solo un $57 \%$ de los entrevistados, están de acuerdo o totalmente de acuerdo en que terminarán el programa académico.

Finalmente, se indagó sobre los niveles de satisfacción con la universidad y sus carreras. Se destaca en los resultados que los estudiantes entrevistados están orgullosos y felices de estudiar en la Universidad Nacional de Colombia; un poco menor es la cantidad de estudiantes felices y orgullosos con sus respectivas carreras, pero en general están satisfechos tanto con la universidad como con sus carreras. Asimismo, y como ítem final, en un $67,5 \%$ creen que la universidad va a mejorar y un $74,5 \%$ opina que sus carreras también lo harán.

Figura No. 7. Satisfacción de los estudiantes de primer semestre

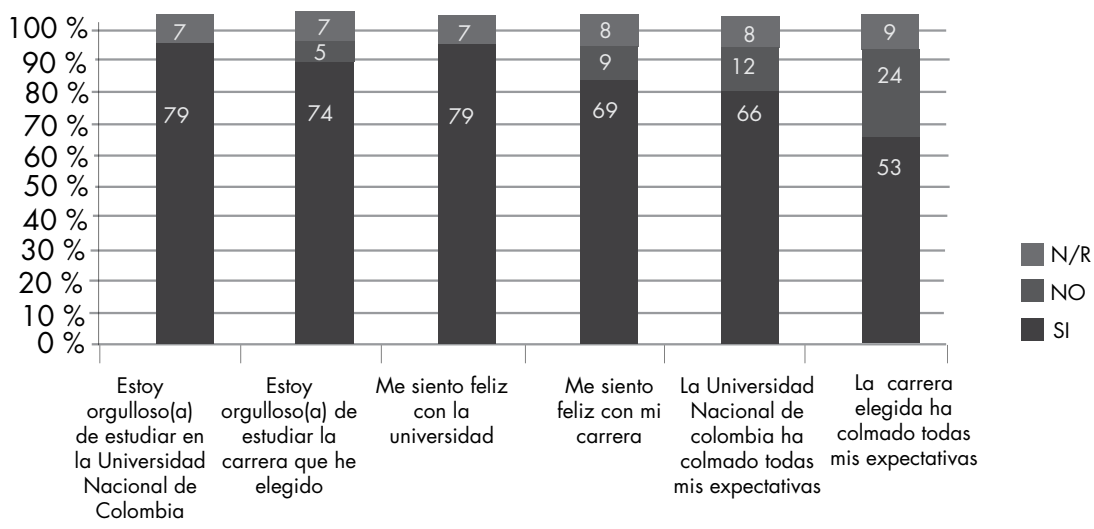

Fuente: La investigación

\section{Conclusiones}

Las motivaciones para la utilización satisfacción derivada de la elección de un servicio pueden ser variadas, puede depender de muchos factores en especial aquellas derivadas de una y es importante para las instituciones necesidad como suele ser la educa- de educación superior reconocer esción secundaria; por lo mismo, la tas necesidades y, a la vez, promover 
la calidad de sus programas vistos por medio del interés de los estudiantes.

La Universidad Nacional ha sido reconocida como la mejor de Colombia; sin embargo, le es importante saber cómo la perciben los aspirantes a ella y posteriormente, los estudiantes de primer semestre, qué los lleva a tomar la decisión de elegirla y establecer si el único criterio es el bajo costo.

Los resultados son alentadores: los recién ingresados piensan que la universidad tiene calidad; es por esto que se les pregunta, no solo por los programas académicos, por los profesores y por los egresados, sino por su percepción de ellos como estudiantes.

En general puede decirse, a partir de la muestra, que los estudiantes encuestados están muy conformes con la universidad y tienen una muy buena imagen de ella, principalmente en su parte académica. Los estudiantes de primer semestre que colaboraron con el ejercicio son conscientes de formar parte de la mejor institución de educación superior nacional, lo que hizo más fácil deducir aspectos como su calidad, la de sus profesores y el buen nombre que conserva y debe preservar. Estas respuestas permiten tener una idea de qué factores se pueden mejorar y cuáles se deben mantener para que la universidad siga siendo lo que es y el estudiante tenga claridad sobre su función, su compromiso y sus responsabilidades al hacer parte de ella.

Estudios como estos resultan de gran utilidad para seguir propiciando los modelos de calidad en la educación superior. Nuevas investigaciones en este sentido deben seguirse realizando, en especial, a la mitad del programa académico, cuando los estudiantes están por graduarse, y finalmente al evaluar su desempeño y percepción como egresados. Asimismo, conocer las expectativas tanto en programas de pregrado como de posgrado. La Universidad Nacional espera seguir aplicando la encuesta a sus estudiantes, no solo a los de la Facultad de Ciencias Económicas, sino a nivel nacional, para conocer dichas expectativas.

\section{Referencias}

Astin, A. (1993). What matters in college? Four critical years revisited. San Francisco: Jossey-Bass.

Biscarri, J., Filelia G. \& Jove, G. (2008). Factores relacionados con la percepción de calidad docente del profesorado universitario. Revista Interuniversitaria de Formación del Profesorado, 20, (3), 287-309. 
Burón, J. (1995). Motivación y aprendizaje. Bilbao: Mensajero.

Caetano, G. (2003). Marketing en los servicios de educación: modelos de percepción de calidad. Madrid: Universidad Complutense de Madrid.

Casero, A. (2010). Factores moduladores de la percepción de la calidad docente. Relieve, 16, (2), 1-17. Recuperado en http://www.uv.es/ relieve/v16n2/relievev16n2_3.htm.

Cole, M. (2003). Cultural Psychology. $A$ once and future discipline ( $5^{\mathrm{a}} \mathrm{Ed}$.). Harvard: Harvard University Press.

Cronin, J. \& TayIor, S. (1994). Serperf vs Servqual: Reconciling perfomance-based and perceptions minus expectations measurement of service quality. Journal of Marketing, 58.

Grönross, C. (1990). Service Management and Marketing. New York: Lexington.

López, R. (2011, 1 de noviembre). Conozca cuáles son las 20 carreras con mejor futuro laboral. La República Bogotá.

López, V. (2004). Evaluación, calificación, credencialismo y formación inicial de profesorado. Revista
Interuniversitaria de Formación del Profesorado, 18, (3), 221-232.

Montoya, A., Castellanos, O. \& Montoya, I (2000). Imagen de la Universidad Nacional de Colombia.Memorias del Simposio internacional de gestión de los procesos universitarios. Pinar del Río, Cuba: Universidad Pinar del Río.

Morales, V. \& Correal, J. (2003). La calidad en la gestión de los servicios deportivos. En A. Hernández Mendo (Coord.). Psicología del deporte (Vol. 3). Aplicaciones. Buenos Aires: Efdeportes.com.

Oliver, R. (1981). A cognitive model of the antecedents and consequences of satisfaction decision. Journal of Marketing Research, 17, 406-409.

(1993). Cognitive, affective, and attribute bases of the satisfaction response. Journal of Consumer Research, 20, 418-430.

Palomero, T. (2004). Europa y calidad docente. Revista Interuniversitaria de Formación del Profesorado, 18, (3), 23-40.

Parasuraman, A., Berry, L.L \& Zeithaml, V.A. (1993). Calidad total en la 
gestión de los servicios. Madrid: Díaz de Santos.

Parasuraman, A., Zeithaml, V.A. \& Berry, L.L. (1988). SERVQUAL: A multiple.item, scale for measuring consumer perceptions of service quality. Journal of Retailing, 64, (1), 12-40.

(1994). Moving Forward in service quality: Measuring different customer-expectation levels, comparing alternative scales, and examining the performance-behavioral intentions link. Cambridge: Marketing Science Institute. (Report Numbers 94-114).

Pichardo, M.C., García, A.B., De la Fuente, J. \& Justicia, F. (2007). El estudio de las expectativas en la universidad: análisis de trabajos empíricos y futuras líneas de investigación. Revista Electrónica de Investigación Educativa, 9, (1).

Prakash, V. (1984). Validity and reliability of confirmation of expectations paradigm. Journal of the Academic of Marketing Science, 12, 63-76.

Rosentahl, R. \& Rubin, D.B. (1978). Interpersonal expectancy effects: the first 345 studies. The Behavioural and Brain Sciences, 3, 377-415.
Rust, R.T. \& Oliver, R.L. (1994). Service quality: Insights and managerial implications from the frontier. En R.T. Rust \& R.L. Oliver (Eds.). Service quality: New directions in Theorie and Practice. Thousand Oaks, California: Sage.

Universidad Nacional de Colombia. (2009). Por una universidad de excelencia, investigadora, innovadora y a la vanguardia del país. Plan global de desarrollo, 2010-2012. Bogotá.

Universidad Nacional de Colombia. (2011). Estadísticas de Admisiones. Recuperado en www.admisiones.unal. edu.co.

Velasco, A. (2011). Variaciones en la percepción de los estudiantes sobre el contexto escolar en universidades públicas y privadas. Boletín Electrónico de Investigación de la Asociación Oaxaqueña de Psicología, 7, (1), 65-76.

Vrontis, D., Thrassou, A. \& Melanthiou, Y. (2007). A contemporary higher education student-choice model for developed countries. Journal of Business Research, 60, 979-989.

Zeithaml, V. (1988). Consumer perceptions of price, quality, and value: a means-end model and synthe- 
- Percepción de la calidad y satisfacción de la elección de los estudiantes - Montoya, L, Montoya, I. \& Rojas, S.

sis of evidence. Journal o/marketing, Zeithaml, V., Parasuraman, A. \& $52,2-22$. Berry, L. (1993). Calidad total en Zeithaml, V., Parasuraman, V. \& Berry, L. (1990). Delivering quality service: Balancing customer perceptions and expectations. Nueva York: la gestión de servicios. Cómo lograr el equilibrio entre las percepciones $y$ las expectativas de los consumidores. Madrid: Díaz de Santos.

The Free Press. 